\title{
Time of day has no effect on maximal aerobic and peak power
}

This article was published in the following Dove Press journal:

ChronoPhysiology and Therapy

9 August 2011

Number of times this article has been viewed

\author{
N Bessot ${ }^{1,3}$ \\ S Moussay ${ }^{1,2}$ \\ B Dufour ${ }^{1,2}$ \\ D Davenne ${ }^{1,2}$ \\ B Sesboüél,3 \\ A Gauthier ${ }^{1,2}$ \\ 'Inserm, ERI27, Caen, France; \\ 'University Caen, Caen, France; \\ ${ }^{3} \mathrm{CHRU}$ Caen, Explorations \\ Fonctionnelles, Caen, France
}

Correspondence: Nicolas Bessot Service de Physiologie, CHU Caen, Université de Caen Basse-Normandie, Av Côte de Nacre,

I4033 Caen Cedex, France

Tel +33231068130

Fax +332 3106 8132

Email bessot-n@phycog.org
Background: The aim of this study was to explore the effect of time of day on peak power reached during an exercise test and maximal aerobic power achieved when the subject reached maximal oxygen uptake.

Methods: Fifteen male competitive endurance cyclists performed a standardized maximal incremental exercise test at 06:00 hours and 18:00 hours. The test began with a 5-minute warmup period at a workload of $150 \mathrm{~W}$. The work rate was then increased by incremental steps of $30 \mathrm{~W}$ per minute until the respiratory exchange ratio reached 1.00 . Thereafter, workload was increased in steps of $15 \mathrm{~W}$ per minute until exhaustion was reached.

Results: No significant diurnal variation was detected in physiological parameters (maximal oxygen uptake and maximal heart rate) or biomechanical parameters (maximal aerobic power, peak power).

Conclusion: Circadian variations classically reported in competitive aerobic performances could be due to fluctuations in maximal aerobic endurance and/or improvement in gestural efficiency (pattern of muscle activity, effective force production, and kinematics).

Keywords: chronobiology, maximal aerobic power, peak power, maximal oxygen uptake, maximal incremental test

\section{Introduction}

It has been demonstrated that physical performance is better in the late afternoon than in the early morning. ${ }^{1}$ For example, maximal performance during $400 \mathrm{~m}$ swim trials has been shown to increase by $2.5 \%$ throughout the day. ${ }^{2}$ Studies focusing on time to exhaustion during fixed high-intensity exercise also demonstrated diurnal variation, with improvement of $9 \%-14 \% .^{3-5}$ The increase in performance observed throughout the day could be partly explained by diurnal fluctuations in both physiological and biomechanical parameters.

Regarding physiological parameters, a number of studies have focused on diurnal fluctuation in aerobic performance determinants, such as maximal heart rate and maximal oxygen uptake. ${ }^{1}$ Hill et $\mathrm{al}^{3}$ and Moussay et $\mathrm{al}^{6}$ did not report any diurnal fluctuation in maximal heart rate. In contrast, other authors ${ }^{7-10}$ have observed a diurnal fluctuation in maximal heart rate of 2-6 beats per minute. There are also large discrepancies between studies that focused on circadian maximal oxygen uptake patterns. Some studies concluded that this variable was stable throughout the day, ${ }^{10-13}$ while others ${ }^{14}$ demonstrated a $4.2 \%$ diurnal variation in this variable.

Both day-to-day biological variability ${ }^{10,11}$ and technical errors in oxygen uptake measurements could mask possible circadian fluctuations in maximal oxygen uptake, 
and could account for differences in the results. When circadian fluctuations are observed in this physiological parameter, the amplitudes reported are rather low and cannot fully explain the diurnal fluctuations in maximal aerobic performance.

Studies designed to explore the rhythmicity of maximal aerobic variables have mainly focused on oxygen uptake fluctuation. However, it is possible that the maximal oxygen uptake measured during a progressive incremental test could be a nearly stable variable while biomechanical parameters such as peak power output reached during the test $\left(\mathrm{P}_{\max }\right)$ and maximal aerobic power, which corresponds to power output measured when the subject reaches maximal oxygen uptake, could vary. In addition, day-to-day biological changes or technical error could result in lower variability in power output measurements compared with oxygen uptake. To our knowledge, diurnal variation in the biomechanical parameters, maximal aerobic power and $\mathrm{P}_{\max }$, have never been investigated. As a consequence, it could be hypothesized that maximal oxygen uptake values collected throughout the day could be constant while maximal aerobic power and/or $\mathrm{P}_{\max }$ could rise from early morning to late afternoon, and thus help to explain the diurnal variations reported in aerobic physical activities. $^{2,3,15}$

A new generation of cycle ergometers allows direct measurement of power output from instantaneous values of crank torque and pedal rate. It seems to be the most accurate and reproducible system for measuring real power output in cycling. ${ }^{16}$ These ergometers function by applying a chosen target power and adjusting the brake in real time according to the subject's instantaneous pedal rate and crank torque. In so doing, the system ensures an accurate measure of power during a maximal aerobic exercise despite the great variability generally observed in pedal rate at the end of the test, when the subject is exhausted.

In order to be valid, an incremental graded test designed to determine maximal aerobic power must be completed within 8-12 minutes. ${ }^{17}$ However, considering the specific case of chronobiological studies, a fluctuation of $2.5 \%-14 \%$ can be expected. ${ }^{1}$ Therefore, the power step increment in the test should be small enough to permit identification of a rhythm.

Taking into account the combination of these constraints, a protocol design for studying maximal aerobic power and $\mathrm{P}_{\text {max }}$ circadian fluctuation should propose power steps that decrease toward the end of the test in order to improve the precision of maximal aerobic power and $\mathrm{P}_{\max }$ measurement. Moreover, to impose the exact power, attention must be paid to the material that is used. The aim of this study was to explore the effect of time of day on maximal aerobic power and $\mathrm{P}_{\max }$ using innovative power meter equipment and an adapted maximal incremental test.

\section{Methods \\ Subjects}

Fifteen well trained male endurance cyclists volunteered to take part in this research (age $19 \pm 2$ years, body weight $65.9 \pm 6.5 \mathrm{~kg}$, height $176.1 \pm 5.3 \mathrm{~cm}$ ). All subjects had had at least 3 years of experience in competitive cycling and usually trained $10.1 \pm 2.6$ hours a week. The study was approved by the ethics committee of CHU Côte de Nacre, Caen, France, and complied with the expectations of the relevant published guidelines. ${ }^{18}$ All subjects provided their written consent once the procedures were explained in detail. The subjects answered the self-assessment questionnaire of Horne and Ostberg, ${ }^{19}$ which determines morningness and eveningness. They were then categorized as either "moderately morning" $(n=5)$ or "neither type" $(n=10)$.

\section{Experimental procedure}

Two test sessions of a maximal incremental test were performed at 06:00 hours and at 18:00 hours on two separate days. The daily timing of the tests corresponded, respectively, with the maximum and minimum of the circadian rhythm of core temperature, oxygen uptake, and heart rate values measured at rest, ${ }^{9-11}$ at submaximal exercise, ${ }^{9,10,14}$ and maximal exercise $^{14}$ for the selected population.

Test sessions were scheduled in a counterbalanced sequence for each subject with a period of at least 36 hours between them. As proposed by Baxter and Reilly, ${ }^{2}$ subjects were awoken at 05:00 hours for the test session at 06:00 hours, being allowed to drink only a glass of water before this session. Their last meal was taken at least 3 hours before the test session at 18:00 hours in order to avoid the effects of postprandial thermogenesis. Subjects were asked to follow their usual sleeping habits as closely as possible with a minimum of 6 hours of sleep ( $6.8 \pm 0.5$ hours) the night preceding each exercise test. They were asked not to train during the 24 hours before each test session. For all tests, the laboratory temperature $\left(20 \pm 1^{\circ} \mathrm{C}\right)$ and humidity $(49 \% \pm 5 \%)$ remained constant in order to minimize environmental influence on muscle function. ${ }^{20}$

During each test session, subjects were asked to ride a cycle ergometer (the SRM High Performance Ergometer ${ }^{\circledR}$, Jülich, Germany) equipped with racing pedals and toe clips. The ergometer was fully adjustable, both vertically and horizontally, to reproduce the exact settings of each subject's bicycle. To avoid the effect of postural changes, ${ }^{21}$ the upper body position was monitored and remained constant 
throughout the exercise. Subjects had their hands in pronation on the upper part of the handlebars and were asked to maintain elbow extension.

The test began with a 5-minute warmup period at a work load of $150 \mathrm{~W}$. The work rate was then increased by $30 \mathrm{~W}$ per minute in steps until the respiratory exchange ratio value reached 1.00. Thereafter, the increasing workload was applied in $15 \mathrm{~W}$ per minute steps until exhaustion was reached (Figure 1). The end of the exercise was determined when the subject could no longer maintain the imposed power output (Figure 1). Throughout each test, the pedal rate was freely chosen by the subject.

\section{Data collection}

Oral temperature was recorded (Thermofina ${ }^{\circledR}$, Dammaries-Le-Lys, France, accuracy $0.1^{\circ} \mathrm{C}$ ) after 15 minutes of rest and before the beginning of the test. For each test session, the power output and pedal rate were continuously monitored using the SRM ergometer system. The "power control" system of the SRM calculated the power output by means of eight constraint gauges located inside the pedals and bottom bracket-bearing assembly in the following way: average torque of a full crank rotation $\times$ average angular velocity of a full crank rotation = average power of a full crank rotation. The instantaneous value of power output was used to adjust the electrobrake in order to correspond to the preprogrammed power. Heart rate, oxygen uptake, and respiratory exchange ratio were recorded continuously by an open-circuit sampling system (K4b², Cosmed ${ }^{\circledR}$, Rome, Italy).

\section{Data analysis}

Each data of heart rate, oxygen uptake, and power output corresponded to a 60 -s mean of raw data. Maximal values were considered as maximal heart rate, maximal oxygen uptake, and $\mathrm{P}_{\max }$, respectively. ${ }^{22}$ To ensure that the maximal oxygen uptake is reached during the exercise, different criteria are generally used. The main criterion is related to the maximal steady state of oxygen uptake which ensures that there is an upper limit on maximal oxygen uptake at the end of exercise despite the increase in power output. The oxygen

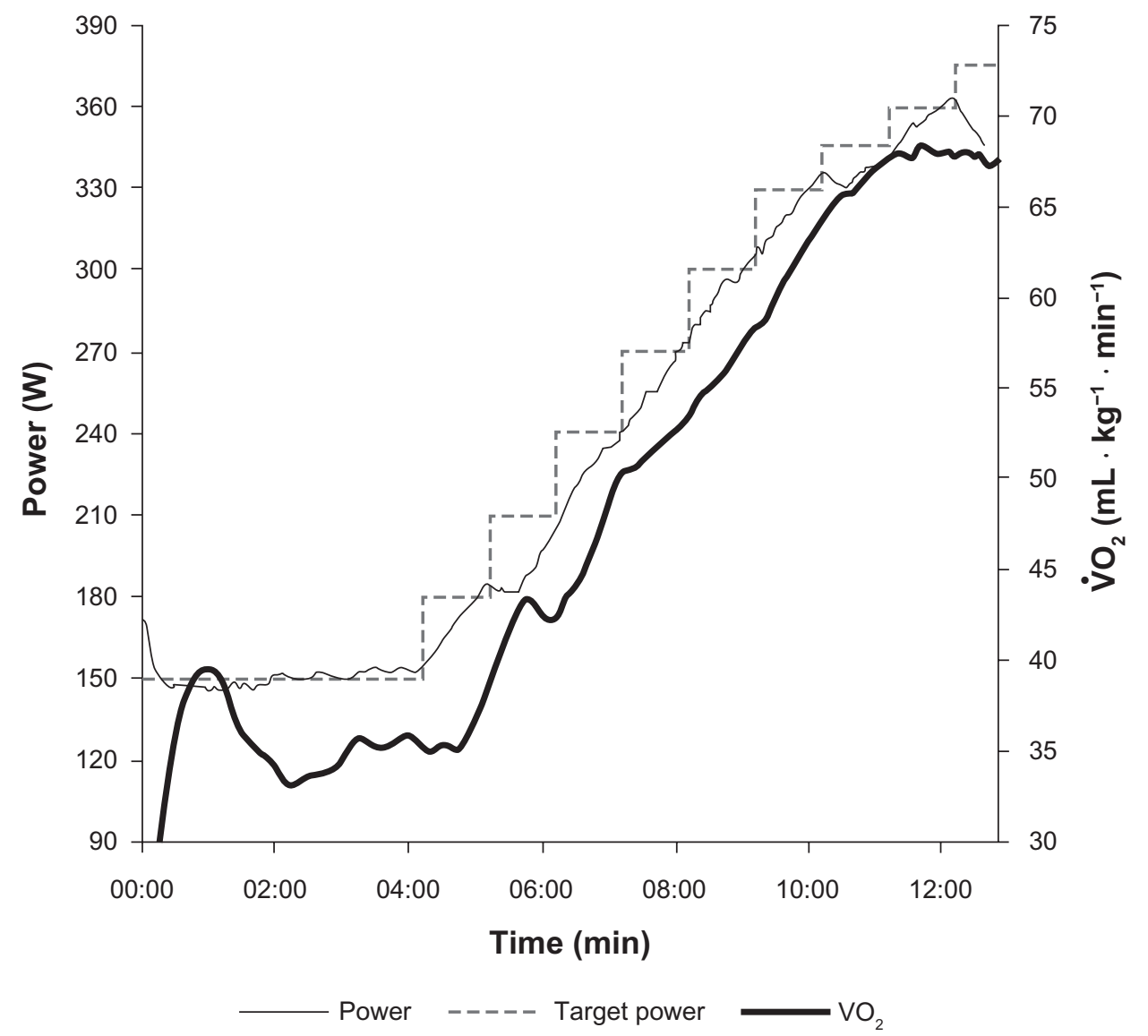

Figure I Representation of test design (subject I, I8:00 hours). Work rate was increased by incremental steps of $30 \mathrm{~W}$ per minute until the respiratory exchange ratio value reached I.00; thereafter, the workload was increased by $15 \mathrm{~W}$ per minute steps until exhaustion was reached. Maximal oxygen uptake and peak power corresponds to the highest values of the 60-s means. Maximal aerobic power was defined as the 60-s mean power output measured at the start of the oxygen uptake plateau. Abbreviation: $\dot{\mathrm{V}}_{2 \max }$, maximal oxygen uptake. 
uptake plateau is attested by the fact that oxygen uptake averaged over the previous stage does not exceed oxygen uptake averaged over the last stage by more than $100 \mathrm{~mL} /$ min. ${ }^{23}$ The next criterion were that the respiratory exchange ratio at the end of the exercise should be greater than 1.10 and heart rate over $90 \%$ of the predicted maximal heart rate. ${ }^{24}$ Maximal aerobic power was defined as the power output measured at the start of the oxygen uptake plateau (maximal oxygen uptake $100 \mathrm{~mL} / \mathrm{min}$, Figure 1).

\section{Statistical analysis}

The normality of distribution of the parameters used in this study was verified using the Kolmogorov Smirnov test. In order to identify time-of-day fluctuations in oral temperature, maximal heart rate, maximal oxygen uptake, $\mathrm{P}_{\max }$, and maximal aerobic power, the values collected for each subject at 06:00 hours and 18:00 hours were compared using the paired $t$-test. Statistical tests were performed using Statistica Software (Statsoft ${ }^{\circledR}$, Tulsa, OK). Statistical significance was accepted at $P<0.05$.

\section{Results}

Resting oral temperature recorded in the late afternoon was significantly higher $\left(35.9^{\circ} \mathrm{C} \pm 0.3^{\circ} \mathrm{C}\right.$ at $06: 00$ hours and $36.6^{\circ} \mathrm{C} \pm 0.3^{\circ} \mathrm{C}$ at $18: 00$ hours) than in the early morning $(P<0.01)$, with a mean increase of $+0.7^{\circ} \mathrm{C} \pm 0.3^{\circ} \mathrm{C}$. For both times of day tested, each subject met all the criteria required to confirm that maximal oxygen uptake was reached at the end of the exercise. Each subject's oxygen uptake value reached a plateau and the maximal respiratory exchange ratio and heart rate mean values collected across test sessions were, respectively, $1.14 \pm 0.05$ and $188 \pm 9$ beats per minute ( $93 \% \pm 2 \%$ of the predicted maximal heart rate).

\section{Physiological parameters}

Maximal oxygen uptake values did not differ between test sessions $(P=0.54)$, and were $63.2 \pm 7.8 \mathrm{~mL} / \mathrm{kg} / \mathrm{min}$ at 06:00 hours and $64.5 \pm 7.2 \mathrm{~mL} / \mathrm{kg} / \mathrm{min}$ at 18:00 hours (Figure 2). Values for maximal heart rate achieved in the late afternoon did not differ $(P=0.10)$ from those observed in the early morning test session $(187.4 \pm 9.8$ beats per minute at 06:00 hours and $190.3 \pm 8.5$ beats per minute at 18:00 hours, Figure 2).

\section{Biomechanical parameters}

$\mathrm{P}_{\max }$ and maximal aerobic power mean values were $332.7 \pm 42.6 \mathrm{~W}$ and $320.2 \pm 41.5 \mathrm{~W}$ at $06: 00$ hours and $342.7 \pm 46.5 \mathrm{~W}$ and $326.2 \pm 43.7 \mathrm{~W}$ at $18: 00$ hours,
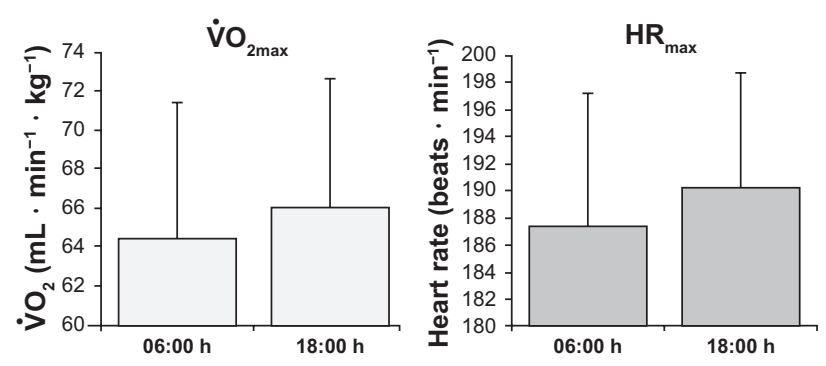

Figure 2 Mean \pm standard deviation maximal oxygen consumption and maximal heart rate recorded at 06:00 hours and 18:00 hours.

Abbreviations: $\dot{\mathrm{V}} \mathrm{O}_{2 \max }$, maximal oxygen uptake; $\mathrm{HR}_{\max }$, maximal heart rate.

respectively (Figure 3), with no difference between test sessions $\left(\mathrm{P}_{\max }[P=0.15]\right.$ or maximal aerobic power $[P=$ $0.22])$.

\section{Discussion}

During the maximal aerobic exercise test, none of the recorded physiological or biomechanical variables showed any significant time of day variation. The experimental design proposed in this study consisted of two test sessions separated by a recovery period of 36-60 hours. Such a protocol design should result in complete recovery between test sessions. Moreover, the short duration of the protocol (one week) should help to avoid the effects of infradian rhythm on the variables collected. ${ }^{25}$ The selected times of day (06:00 hours and 18:00 hours) fit with the usual trough and peak of the circadian rhythm of physical performance and oral temperature reported in this type of population..$^{10,11}$

The resting oral temperature demonstrates a significant $0.7^{\circ} \mathrm{C}$ rise from $06: 00$ hours to $18: 00$ hours. This range of variation is in good accordance with the maximal circadian difference in oral temperature usually reported for physically active subjects. ${ }^{19,26,27}$ Considering that performancedetermining factors and core temperature circadian variations are roughly in phase, ${ }^{1,9}$ it can be assumed that the present study should have allowed us to observe the maximal

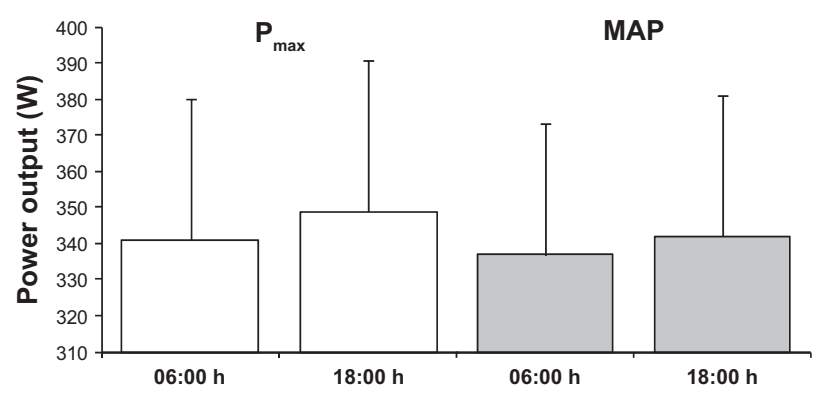

Figure 3 Mean \pm standard deviation peak power and maximal aerobic power recorded at 06:00 hours and 18:00 hours. 
amplitude of circadian variation in recorded variables. In an effort to complete the literature related to time of day effects on maximal aerobic performance, this study used original recorded variables and test design as well as instrumentation not seen in previous chronobiological studies.

The results of the present study failed to demonstrate any significant diurnal variation in maximal heart rate and maximal oxygen uptake. Reilly et $\mathrm{al}^{8}$ showed that amplitude of the circadian rhythm in heart rate is reduced as exercise intensity increases. Therefore, some discrepancies regarding the existence of diurnal fluctuation of maximal heart rate exist in the literature. Hill et $\mathrm{al}^{3}$ and Moussay et $\mathrm{al}^{6}$ did not report any maximal heart rate diurnal fluctuation. However, other authors have observed a diurnal fluctuation in maximal heart rate..$^{7-10}$ The maximal heart rate diurnal fluctuation previously observed in these studies ranges from 2 to 6 beats per minute (mean 1\%-3\%). Considering our data, the difference between mean test session values $(2.9 \pm 6.4$ beats per minute $)$ fit with such ranges of variation but failed to be significant, perhaps because of the large variability between subjects.

Many authors have already attempted to explore circadian variation in maximal oxygen uptake. Indeed, Faria and Drummond ${ }^{12}$ programmed test sessions at 12 different times of day and duplicated measurements at each time point. Reilly and Brooks ${ }^{10,11}$ used a longitudinal design to avoid variability between subjects, as well as a cross-sectional approach. The day-to-day coefficient of variation of maximal oxygen uptake was found to be $2.9 \%$. Considering the low hypothetical fluctuation in amplitude of maximal oxygen uptake during the day, if the interday variability and accuracy of oxygen uptake measurement equipment is taken into account, ${ }^{28}$ it is not surprising that all these studies failed to observe circadian variation in maximal oxygen uptake, which is confirmed by the present study. However, these results, which were somewhat expected, could have been associated with some variation in biomechanical parameters.

In order to identify a time-of-day fluctuation in maximal aerobic power and $\mathrm{P}_{\max }$, we used an innovative test design and improved materials. The increment proposed in the last steps of the present design was lower than in other protocols that focused on circadian rhythmicity. ${ }^{6,14}$ The $15 \mathrm{~W}$ steps used at the end of the test correspond to $4.5 \%$ of mean maximal aerobic power, which is close to the expected diurnal variation of this variable. Moreover, the SRM ergometer used in the present study allows direct measurement of crank power $(1 \mathrm{~Hz})$. Therefore, it could be hypothesized that the power recorded during exercise was the power that was actually developed against external resistance while taking into account the inertia of the ergometer and the delay (around 10 seconds) in adjustment of the brake due to pedal rate variation (compare with Figure 1).

Despite the improvement in accuracy made possible in this original protocol design, no significant diurnal variation was detected in maximal aerobic power and $\mathrm{P}_{\max }$. Maximal power achieved during maximal aerobic tests, such as maximal oxygen uptake, did not fluctuate during the two times of day tested, so could not explain diurnal variation in aerobic performance. ${ }^{2,3,15}$ However, diurnal variation in muscle function has previously been reported. Peak muscular torque $^{29,30}$ and fatigability ${ }^{31}$ increase throughout the day. Nerve conduction velocity also increases throughout the day $^{32}$ and time to peak and relaxation times decrease by $8.3 \%$ and $10.7 \%$, respectively, ${ }^{30}$ making for better muscle properties in the late afternoon than in the early morning. Therefore, it seems that diurnal changes in muscle function do not improve the efficiency of the biomechanical parameters tested in this study.

With regard to the limitations of our present research, using only two measurements daily was not optimal for highlighting circadian variations. However, the selected times of day fit with the usual troughs and peaks of circadian rhythm in physical performance. However, some delay may exist between subjects and the variables measured. The incremental exercise performed by the subjects reaches exhaustion and the motivation level of the subjects determines the reliability of the result, so we chose to limit the number of test sessions to preserve the subjects' motivation.

Regarding methodological restrictions, the sample size tested may be a determining factor in demonstrating a significant diurnal fluctuation in maximal aerobic variables. Hill et al, ${ }^{14}$ who observed a mean variation of $4.1 \%$ in maximal oxygen uptake, included twice as many subjects in their study as in the present one. A greater number of subjects could have been included to achieve statistical significance and/or increase the statistical power $(<0.25$ for each analysis in this study). Based on the size effect determined by the results of this study, a statistical power of 0.8 could be achieved with either 50 subjects for maximal aerobic power or 100 subjects for maximal oxygen consumption. The rather small time of day difference and the high standard deviation observed in this study imply the need to include more subjects to confirm our results with more statistical certainty. Moreover, the nonsignificant $3 \%$ and $1 \%$ differences, observed for mean maximal aerobic power and $\mathrm{P}_{\max }$, respectively, were not consistent with the minimal $9 \%$ diurnal variation previously reported in maximal aerobic performance. ${ }^{3-5}$ 
In conclusion, the diurnal fluctuations commonly reported in maximal aerobic performance can neither be explained by fluctuations in physiological parameters (maximal heart rate and maximal oxygen uptake) nor by diurnal variations in biomechanical parameters (maximal aerobic power and $\mathrm{P}_{\max }$ ). These fluctuations could instead be explained by fluctuations in maximal aerobic endurance and/or improvement in gestural efficiency throughout the day. Indeed, as previously observed, time to exhaustion increases from 06:00 hours to 18:00 hours, ${ }^{4,5,15}$ and the pattern of muscle activity, effective force production, and kinematics during cycling are dependent on time of day. ${ }^{33,34}$ This hypothesis should be tested in further research.

\section{Disclosure}

The authors report no conflicts of interest in this work.

\section{References}

1. Reilly T. Human circadian rhythms and exercise. Crit Rev Biomed Eng. 1990;183:165-180.

2. Baxter C, Reilly $\mathrm{T}$. Influence of time of day on all out swimming. Br J Sports Med. 1983;17:122-127.

3. Hill DW, Borden DO, Darnaby KM, Hendricks DN, Hill CM. Effect of time of day on aerobic and anaerobic responses to high-intensity exercise. Can J Sports Sci. 1992;17:316-319.

4. Hill DW. Effect of time of day on aerobic power in exhaustive high-intensity exercise. J Sports Med Phys Fitness. 1996;36:155-160.

5. Bessot N, Nicolas A, Moussay S, Gauthier A, Sesboüé B, Davenne D. The effect of pedal rate and time of day on the time to exhaustion from high-intensity exercise. Chronobiol Int. 2006;23:1009-1024.

6. Moussay S, Gauthier A, Sesboüé B, Davenne D. Effets de l'heure de la journée sur les paramètres physiologiques et biomécaniques de la performance en cyclisme. Sciences et Motricité. 2002;46:95-117.

7. Yamaji K, Sakamoto H, Nakaguchi M, Kitamura K, Shepard RJ. Biological rhythms of PWC170 and maximal oxygen uptake. J Hum Ergol. 1981;10:213-219.

8. Reilly T, Robinson G, Minors DS. Some circulatory responses to exercise at different times of day. Med Sci Sports Exerc. 1984;16:477-482.

9. Cable NT, Reilly T. Influence of circadian rhythms on arm exercise. J Hum Mov Studies. 1987;13:13-17.

10. Reilly T, Brooks GA. Selective persistence of circadian rhythms in physiological responses to exercise. Chronobiol Int. 1990;7:59-67.

11. Reilly T, Brooks GA. Investigation of circadian rhythms in metabolic responses to exercise. Ergonomics. 1982;25:1093-1107.

12. Faria IE, Drummond BJ. Circadian changes in resting heart rate and body temperature, maximal oxygen consumption and perceived exertion. Ergonomics. 1982;25:381-386.

13. Deschenes MR, Sharma JV, Brittingham KT, Casa DJ, Armstrong LE, Maresh CM. Chronobiological effects on exercise performance and selected physiological responses. Eur J Appl Physiol Occup Physiol. 1998;77:249-256.

ChronoPhysiology and Therapy

\section{Publish your work in this journal}

ChronoPhysiology and Therapy is an international, peer-reviewed, open access journal focusing on research into the cyclic variations and rhythmicity in physiological processes in the body and the research and development and optimal timing of administration of therapeutic targets to achieve improved outcomes and quality of life for the patient. The
14. Hill DW, Cureton KJ, Collins MA, Grisham SC. Diurnal variations in responses to exercise of "morning types" and "evening types". J Sports Med Phys Fitness. 1988;283:213-219.

15. Reilly T, Baxter C. Influence of time of day on reactions to cycling at a fixed high intensity. Br J Sports Med. 1983;17:128-130.

16. Balmer J, Davison RDR, Bird SE. Reliability of an air-braked ergometer to record peak power during a maximal cycling test. Med Sci Sports Exerc. 2000;10:1790-1793.

17. Buchfuhrer MJ, Hansen JE, Robinson TE, Sue DY, Wasserman K, Whipp BJ. Optimizing the exercise protocol for cardiopulmonary assessment. J Appl Physiol. 1983;55:1558-1564.

18. Portaluppi F, Touitou Y, Smolensky MH. Ethical and methodological standards for laboratory and medical biological rhythm research. Chronobiol Int. 2008;25:999-1016.

19. Horne JA, Östberg O. A self-assessment questionnaire to determine morningness-eveningness in human circadian rhythms. Int J Chronobiol. 1976;4:97-110.

20. Bergh U, Ekblom B. Influence of muscle temperature on maximal muscle strength and power output in human skeletal muscles. Acta Physiol Scand. 1979;107:33-37.

21. Clarys JP, Alewaeters K, Zinzen E. The influence of geographic variations on the muscular activity in selected sports movements. J Electromyogr Kinesiol. 2001;11:451-457.

22. Hill DW, Poole DC, Smith JC. The relation between power and the time to achieve $\dot{V}_{2_{\max }}$. Med Sci Sports Exerc. 2002;344:709-714.

23. Di Prampero PE, Capelli D, Pagliaro P. Energetics of endurance in middle-distance running. Eur J Appl Physiol. 1993;55:259-266.

24. Astrand PO, Rodahl K. Textbook of work physiology. New York, NY: McGraw-Hill; 1986.

25. Halberg F, Reinberg A. Circadian frequency and low frequency rhythms in human physiology. $J$ Physiol (Paris). 1967;59(Suppl 1):117-120. French.

26. Ilmarinen J, Ilmarinen R, Korhonen O, Nurminen M. Circadian variation of physiological functions related to physical work capacity. Scand J Work Environ Health. 1980;6:112-122.

27. Reilly T, Down A. Investigation of circadian rhythms in anaerobic power and capacity of the legs. J Sports Med Phys Fitness. 1992;32:343-347.

28. McLaughlin JE, King GA, Howley ET, Bassett DR Jr, Ainsworth BE. Validation of the COSMED K4 b2 portable metabolic system. Int $J$ Sports Med. 2001;22:280-284.

29. Gauthier A, Davenne D, Gentil C, Van Hoecke J. Circadian rhythm in the torque developed by elbow flexors during isometric contraction, effect of sampling schedules. Chronobiol Int. 1997;14:287-294.

30. Martin A, Carpentier A, Guissard N, Van Hoecke J, Duchateau J. Effect of time of day on force variation in a human muscle. Muscle Nerve. 1999;22:1380-1387.

31. Nicolas A, Gauthier A, Bessot N, Moussay S, Davenne D. Time-of-day effects on myoelectric and mechanical properties of muscle during maximal and prolonged isokinetic exercise. Chronobiol Int. 2005;22:997-1011.

32. Ferrario VF, Tredici G, Crespi V. Circadian rhythm in human nerve conduction velocity. Chronobiologia. 1980;7:205-209.

33. Bessot N, Moussay S, Clarys JP, Gauthier A, Sesboüé B, Davenne D. The influence of circadian rhythm on muscle activity and efficient force production during cycling at different pedal rates. J Electromyogr Kinesiol. 2007;17:176-183.

34. Moussay S, Bessot N, Gauthier A, Larue J, Sesboüe B, Davenne D. Diurnal variations in cycling kinematics. Chronobiol Int. 2003;20:879-892.

\section{Dovepress}

manuscript management system is completely online and includes a very quick and fair peer-review system. Visit http://www.dovepress.com/ testimonials.php to read real quotes from published authors. 